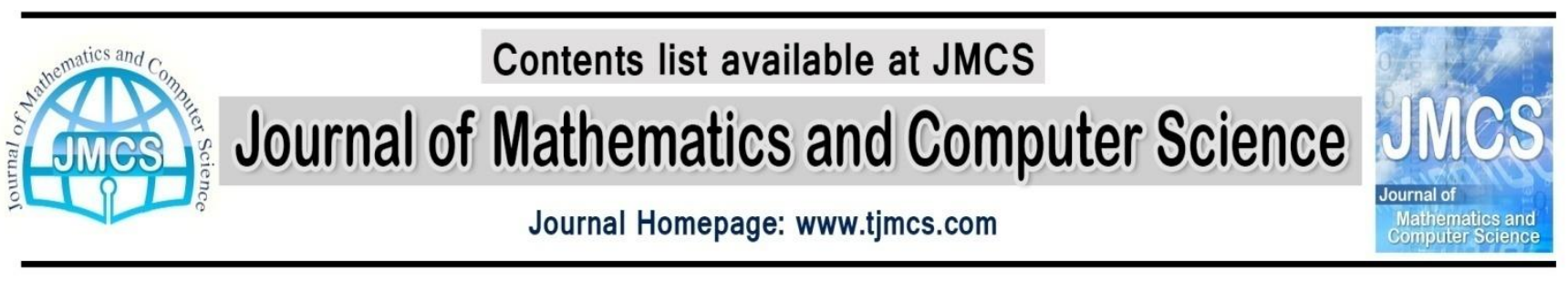

\title{
A numerical method for space fractional diffusion equations using a semi- disrete scheme and Chebyshev collocation method
}

\author{
Hadi Azizi ${ }^{1}$, Ghasem Barid Loghmani ${ }^{2}$ \\ ${ }^{1}$ Department of Mathematics, Taft Branch, Islamic Azad University, Taft, Iran \\ Hadiazizi1360@yahoo.com \\ ${ }^{2}$ Department of Mathematics, Yazd University, Yazd, Iran \\ Loghmani@yazduni.ac.ir
}

Article history:

Received June 2013

Accepted July 2013

Available online July 2013

\begin{abstract}
In the present paper, a numerical approach to efficiently calculate the solution of space fractional diffusion equations is investigated. The finite difference scheme and Chebyshev collocation method is applied to solve this problems. Also, the matrix form of the proposed method is obtained. The numerical examples and comparison with other methods shows that the present method is effective.
\end{abstract}

Keywords: Fractional diffusion equation, Finite difference, Collocation, Chebyshev polynomials

\section{Introduction}

The use of fractional partial differential equations (FPDEs) in mathematics, physics, engineering and chemistry has become increasingly popular in recent years $[4,8,14]$.

To obtain an analytical solution of this problems is extremely difficult thus many authors are seeking ways to numerically solve these problems.

Some different numerical methods have been proposed for solving FPDEs. Liu and et al. proposed method of lines to transform the space fractional Fokker-Planck equation into a system of ordinary differential equations [7]. Fix and Roop [5] developed a least squares finite element solution of a fractional-order two-point boundary value problems. Afrouzi and et al. [1] proposed Homotopy-perturbation method for a kind of Time-Fractional Evolution Equations. In [3] Darzi and et al. used Sumudu transform method for solving fractional differential equations and fractional Diffusion-Wave equation. Also Neamaty [13] solved Fractional Partial Differential Equation by Using Wavelet Operational Method.

Meerschaert, Tadjeran and et al. suggested three kinds of finite difference approximations which are the implicit Euler method, the explicit Euler method and the fractional Cranck-Nicholson method 
for FPDEs based on shifted Grünwald formula. Also they derived some detailed stability and convergence analysis $[10,11,12,18,19]$.

The space fractional diffusion equations are a type of fractional partial differential equations which by many authors are solved numerically. For example Khader [6] used Chebyshev collocation method to discretize space fractional diffusion equations to obtain a linear system of ordinary differential equations and used the finite difference method for solving the resulting system. Saadatmandi and Dehghan [15] used tau approach and Sousa [16] applied splines to solve space fractional diffusion equations.

In this paper, we proposed a different approach to obtain the solution of space fractional diffusion equation

$$
\frac{\partial u(x, t)}{\partial t}=p(x) \frac{\partial^{\alpha} u(x, t)}{\partial x^{\alpha}}+v(x, t), 0<x<1,0 \leq t \leq T, 1<\alpha \leq 2,
$$

with initial condition

$$
u(x, 0)=g(x), 0<x<1,
$$

and boundary conditions

$$
\begin{aligned}
& u(0, t)=h_{0}(t), 0<t \leq T, \\
& u(1, t)=h_{1}(t), 0<t \leq T .
\end{aligned}
$$

Where the function $v(x, t)$ is a source term and the fractional derivative is the Caputo derivative can be defined by [16]:

$$
\frac{\partial^{\alpha} u(x, t)}{\partial x^{\alpha}}=\frac{1}{\Gamma(m-\alpha)} \int_{0}^{x}(x-s)^{m-\alpha-1} \frac{\partial^{m} u(s, t)}{\partial s^{m}} d s, m-1<\alpha \leq m .
$$

Note that for $\alpha=2$, Eq.(1) is the classical diffusion equation

$$
\frac{\partial u(x, t)}{\partial t}=p(x) \frac{\partial^{2} u(x, t)}{\partial x^{2}}+v(x, t) .
$$

This work presents a numerical method to solve this kind of problems using finite difference scheme and collocation method via Chebyshev polynomials.

\section{Description of the method}

In this section, the process of solving the space fractional diffusion equations is described as in 1-4.

Let $t_{n}=n \Delta t, n=0,1, \ldots, M$ where $\Delta t=\frac{T}{M}, t_{0}=0, t_{M}=T$.

First, we use a finite difference technique and $\theta$-weighted scheme [2] to discritize the time derivative.

$$
\frac{u(x, t+\Delta t)-u(x, t)}{\Delta t}=\frac{p(x)}{\Gamma(2-\alpha)} \int_{0}^{x}(x-s)^{1-\alpha}\left[\theta \frac{d^{2} u(s, t+\Delta t)}{d s^{2}}+(1-\theta) \frac{d^{2} u(s, t)}{d s^{2}}\right] d s+v(x, t),
$$

here $\theta \in[0,1]$. Using the notation $u_{n}(x)=u\left(x, t_{n}\right)$ in (5), where $t_{n}=t_{n-1}+\Delta t$, we obtain

$$
\begin{aligned}
u_{n+1}(x)-u_{n}(x) & =p(x) \frac{\Delta t}{\Gamma(2-\alpha)} \int_{0}^{x}(x-s)^{1-\alpha}\left[\theta \frac{d^{2} u_{n+1}(s)}{d s^{2}}\right. \\
& \left.+(1-\theta) \frac{d^{2} u_{n}(s)}{d s^{2}}\right] d s+\Delta t v_{n}(x), n=0,1, \ldots, M-1,
\end{aligned}
$$

where $v_{n}(x)=v\left(x, t_{n}\right)$. 
Definition 1. [9] The well-known Chebyshev polynomials of the first kind of degree $n$ are defined on the interval $[-1,1]$ as

$$
T_{n}(x)=\cos (n \arccos (x)),
$$

where $T_{0}(x)=1, T_{1}(x)=x$ and they satisfy the recurrence relations:

$$
T_{n+1}(x)=2 x T_{n}(x)-T_{n-1}(x), n=1,2, \cdots .
$$

In order to use these polynomials on the interval $[0,1]$ we define the so called shifted Chebyshev polynomials by introducing the change of variable $z=2 x-1$. The shifted Chebyshev polynomials are defined as: $T_{n}^{*}(x)=T_{n}(2 x-1)$.

Now we expand $u_{n}(x)$ by shifted Chebyshev polynomials:

$$
u_{n}(x)=\sum_{i=0}^{N} r_{i}^{n} T_{i}^{*}(x), n=1, \ldots, M,
$$

where $r_{0}^{n}, r_{1}^{n}, \ldots, r_{N}^{n}$ are unknown coefficients.

From equation (6) and (7) we obtain:

$$
\begin{aligned}
\sum_{i=0}^{N} r_{i}^{n+1} T_{i}^{*}(x)-\sum_{i=0}^{N} r_{i}^{n^{*}}(x) & =p(x) \frac{\Delta t}{\Gamma(2-\alpha)} \int_{0}^{x}(x-s)^{1-\alpha}\left[\theta \sum_{i=0}^{N} r_{i}^{n+1} T_{i}^{*^{* \prime}}(s)\right. \\
& \left.+(1-\theta) \sum_{i=0}^{N} r_{i}^{n^{* *^{\prime \prime}}}(s)\right] d s+\Delta t v_{n}(x), \quad n=0,1, \ldots, M-1 .
\end{aligned}
$$

In order to find the unknown coefficients, Chebyshev collocation method with collocation points $x_{k}=\frac{1}{2} \cos \left(\frac{k \pi}{N}\right)+\frac{1}{2}, k=0,1, \ldots, N$ are applied.

$$
\begin{gathered}
\sum_{i=0}^{N} r_{i}^{n+1} T_{i}^{*}\left(x_{k}\right)-\sum_{i=0}^{N} r_{i}^{n^{*}}{ }_{i}\left(x_{k}\right)=p\left(x_{k}\right) \frac{\Delta t}{\Gamma(2-\alpha)} \int_{0}^{x_{k}}\left(x_{k}-s\right)^{1-\alpha}\left[\theta \sum_{i=0}^{N} r_{i}^{n+1} T_{i}^{*^{\prime \prime}}(s)+(1-\theta) \sum_{i=0}^{N} r_{i}^{n^{*^{\prime \prime}}}(s)\right] d s \\
+\Delta t v_{n}\left(x_{k}\right), n=0, \ldots, M-1, k=1, \ldots, N-1 .
\end{gathered}
$$

Also boundary conditions ( 3 ) and (4) for $n=0,1, \ldots, M-1$ are used to obtain:

$$
\begin{gathered}
u_{n+1}\left(x_{N}\right)=\sum_{i=0}^{N} r_{i}^{n+1^{*}}\left(x_{N}\right)=h_{0}\left(t_{n+1}\right), \\
u_{n+1}\left(x_{0}\right)=\sum_{i=0}^{N} r_{i}^{n+1^{*}}\left(x_{0}\right)=h_{1}\left(t_{n+1}\right) .
\end{gathered}
$$

Therefor eqautions (9)-(11) generate a set of $(\mathrm{N}+1)$ algebraic equations, which can be solved for unknown coefficients.

Clearly $u_{0}(x)$ can be obtained from the initial condition (2) as follows:

$$
u_{0}(x)=u\left(x, t_{0}\right)=g(x)
$$

\section{The matrix form of the proposed method}

In order to find the matrix form of suggested method, first by using (9) for $k=1,2, \ldots, N-1$ we obtain the preliminary matrices, $\mathbf{T}^{*}$ and $\mathbf{Q}$. Finally by using (10) and (11) the matrix form for this method is

Atchieved.

$$
\text { Let }[u]^{n}=\left[u_{1}^{n}, u_{2}^{n}, \ldots, u_{N-1}^{n}\right]^{T} \text { and }[r]^{n}=\left[r_{0}^{n}, r_{1}^{n}, \ldots, r_{N}^{n}\right]^{T} \text { where } u_{k}^{n}=u_{n}\left(x_{k}\right), k=1,2, \ldots, N-1 \text {. }
$$


From (7) we obtain

$$
[u]^{n}=\mathbf{T}^{*}[r]^{n}
$$

where $\mathbf{T}^{*}$ is a $(N-1) \times(N+1)$ matrix that given by

$$
\mathbf{T}^{*}=\left[\begin{array}{ccc}
T_{0}^{*}\left(x_{1}\right) & T_{1}^{*}\left(x_{1}\right) & T_{N}^{*}\left(x_{1}\right) \\
T_{0}^{*}\left(x_{2}\right) & T_{1}^{*}\left(x_{2}\right) & T_{N}^{*}\left(x_{2}\right) \\
& & \\
T_{0}^{*}\left(x_{N-1}\right) & T_{1}^{*}\left(x_{N-1}\right) & T_{N}^{*}\left(x_{N-1}\right)
\end{array}\right]
$$

Also from the right side of equations (6) and (9) for $k=1,2, \ldots, N-1$ we obtain

$$
\begin{gathered}
\frac{p\left(x_{k}\right) \Delta t}{\Gamma(2-\alpha)} \int_{0}^{x_{k}}\left(x_{k}-s\right)^{1-\alpha}\left[\theta \frac{d^{2} u_{n+1}(s)}{d s^{2}}+(1-\theta) \frac{d^{2} u_{n}(s)}{d s^{2}}\right] d s+\Delta t v_{n}\left(x_{k}\right) \\
=\frac{p\left(x_{k}\right) \Delta t}{\Gamma(2-\alpha)} \int_{0}^{x_{k}}\left(x_{k}-s\right)^{1-\alpha}\left[\theta \sum_{i=0}^{N} r_{i}^{n+1} T_{i}^{*^{\prime \prime}}(s)+(1-\theta) \sum_{i=0}^{N} r_{i}^{n} T_{i}^{*^{\prime \prime}}(s)\right] d s+\Delta t v_{n}\left(x_{k}\right) \\
=\theta \Delta t \sum_{i=0}^{N} r_{i}^{n+1}\left(\frac{p\left(x_{k}\right)}{\Gamma(2-\alpha)} \int_{0}^{x_{k}}\left(x_{k}-s\right)^{1-\alpha} T_{i}^{*^{\prime \prime}}(s) d s\right)+(1-\theta) \Delta t \sum_{i=0}^{N} r_{i}^{n}\left(\frac{p\left(x_{k}\right)}{\Gamma(2-\alpha)} \int_{0}^{x_{k}}\left(x_{k}-s\right)^{1-\alpha} T_{i}^{*^{\prime \prime}}(s) d s\right) \\
+\Delta t v_{n}\left(x_{k}\right) .
\end{gathered}
$$

Therefor the matrix form for this equations is as follows:

$$
\theta \Delta t \mathbf{Q}[r]^{n+1}+(1-\theta) \Delta t \mathbf{Q}[r]^{n}+\Delta t \mathbf{v}_{\mathbf{n}},
$$

where

$$
\mathbf{v}_{\mathbf{n}}=\left[v_{n}\left(x_{1}\right), v_{n}\left(x_{2}\right), \ldots, v_{n}\left(x_{N-1}\right)\right]^{T} .
$$

and the matrix element of $\mathbf{Q}$ are

$$
q_{i j}=\frac{p\left(x_{i}\right)}{\Gamma(2-\alpha)} \int_{0}^{x_{i}}\left(x_{i}-s\right)^{1-\alpha} T_{j-1}^{*}{ }^{\prime \prime}(s) d s,
$$

for $i=1, \ldots, N-1, j=1, \ldots, N+1$.

Since

$$
D_{*}^{\alpha} T_{j}^{*}(x)=\frac{1}{\Gamma(2-\alpha)} \int_{0}^{x}(x-s)^{1-\alpha} T_{j}^{*^{\prime \prime}}(x) d s, 1<\alpha \leq 2,
$$

and from [6] we have:

$$
D_{*}^{\alpha} T_{j}^{*}(x)=\sum_{k=0}^{j-[\alpha]}(-1)^{k} 2^{2(j-k)} \frac{j(2 j-k-1) !(j-k) !}{k !(2 j-2 k) ! \Gamma(j-k+1-\alpha)} x^{j-k-\alpha},
$$

thus the matrix elements of $\mathbf{Q}$ can be demonstrated as follows:

$$
q_{i j}=\left\{\begin{array}{cc}
0, & 1 \leq i \leq N-1, j=1,2, \\
p\left(x_{i}\right) \sum_{k=0}^{j-[\alpha]-1}(-1)^{k} 2^{2(j-k-1)} \frac{(j-1)(2 j-k-3) !(j-k-1) !}{k !(2 j-2 k-2) ! \Gamma(j-k-\alpha)} x_{i}^{j-k-\alpha-1}, & 1 \leq i \leq N-1,3 \leq j \leq N+1 .
\end{array}\right.
$$

Note that $D_{*}^{\alpha} T_{j}^{*}(x)=0$ for $j=0,1, \ldots,[\alpha]-1$ thus $q_{i j}=0$ for $\mathrm{j}=1,2$.

Therefore above matrices and equations (9) for $k=1,2, \ldots, N-1$ leads to the following matrix form:

$$
\left(\mathbf{T}^{*}-\theta \Delta t \mathbf{Q}\right)[r]^{n+1}=\left((1-\theta) \Delta t \mathbf{Q}+\mathbf{T}^{*}\right)[r]^{n}+\Delta t \mathbf{v}_{\mathbf{n}} .
$$

Now by using boundary conditions (10), (11) and relation (12) we obtain the matrix form of our method as follows: 


$$
\mathbf{A}[r]^{n+1}=\mathbf{B}[r]^{n}+\mathbf{d}_{\mathbf{n}}
$$

where the matrix elements of $\mathbf{A}$ are:

$$
a_{i j}= \begin{cases}T_{j-1}^{*}\left(x_{0}\right), & i=1,1 \leq j \leq N+1, \\ T_{j-1}^{*}\left(x_{N}\right), & i=N+1,1 \leq j \leq N+1, \\ T_{j-1}^{*}\left(x_{i-1}\right), & 2 \leq i \leq N, j=1,2, \\ T_{j-1}^{*}\left(x_{i-1}\right)-\theta \Delta t p\left(x_{i}\right) \sum_{k=0}^{j-[\alpha]-1}(-1)^{k} 2^{2(j-k-1)} \frac{(j-1)(2 j-k-3) !(j-k-1) !}{k !(2 j-2 k-2) ! \Gamma(j-k-\alpha)} x_{i-1}^{j-k-\alpha-1}, & 2 \leq i \leq N, 3 \leq j \leq N+1,\end{cases}
$$

matrix $\mathbf{B}$ is obtained by adding two zero rows to the first and last row of matrix $\left((1-\theta) \Delta t \mathbf{Q}+\mathbf{T}^{*}\right)$ and

$$
\mathbf{d}_{\mathbf{n}}=\left[h_{1}\left(t_{n+1}\right), \Delta t \mathbf{v}_{\mathbf{n}}{ }^{T}, h_{0}\left(t_{n+1}\right)\right]^{T} .
$$

\section{Numerical examples}

In this section, we consider some examples of proposed scheme for the space fractional diffusion equations and for showing the accuracy, efficiency and validity of the method, we compare the our numerical results with other methods.

Remark: In all of examples the time step is taken as $\Delta t=0.001$ and $\theta=\frac{1}{2}$.

Example 4.1. [15] Consider the following space fractional diffusion equation

$$
\frac{\partial u(x, t)}{\partial t}=p(x) \frac{\partial^{1.5} u(x, t)}{\partial x^{1.5}}+v(x, t)
$$

on a finite domain $0<x<1$, with the diffusion coefficient

$p(x)=\Gamma(1.5) x^{0.5}$,

the source function

$v(x, t)=\left(x^{2}+1\right) \cos (t+1)-2 x \sin (t+1)$,

with the initial condition

$u(x, 0)=\left(x^{2}+1\right) \sin (1)$,

and the boundary conditions

$u(0, t)=\sin (t+1), \quad u(1, t)=2 \sin (t+1), \quad$ for $t>0$.

The exact solution of this problem is $u(x, t)=\left(x^{2}+1\right) \sin (t+1)$.

We applied the present method with $N=2$ and compared absolute error function $\left|u(x, 1)-u_{\text {approx }}(x, 1)\right|$ of our scheme with the method in [15] which are shown in Table 1. In Table 2 the maximum absolute errors for $t=0,0.1,0.2, \ldots, 0.9,1$ and $0<x<1$ are reported.

Also, figure 1 shows the exact solution and approximate solution for $\mathrm{u}(\mathrm{x}, 1)$ with $N=2$. Note that the matrices $\mathbf{A}$ and $\mathbf{B}$ for this example are as follows:

$$
\begin{aligned}
A & =\left[\begin{array}{ccc}
1 & 1 & 1 \\
1 & 0 & -1.002 \\
1 & -1 & 1
\end{array}\right], \\
B & =\left[\begin{array}{ccc}
0 & 0 & 0 \\
1 & 0 & -0.998 \\
0 & 0 & 0
\end{array}\right] .
\end{aligned}
$$


Easily seen that $\mathbf{A}$ is non-singular matrix and the spectral radius of matrix $\mathbf{A}^{-1} \mathbf{B}$ is less than one therefore the proposed method has unique solution and is unconditionally stable [17].

Table 1: Comparison of present method for $u(x, 1)$ with the tau method [15] for Exa. 4.1.

\begin{tabular}{ccc}
\hline$x$ & Method[15] with $\mathrm{m}=7$ & present method with $\mathrm{N}=2$ \\
\hline 0.1 & $4.66 \times 10^{-5}$ & $9.34 \times 10^{-7}$ \\
0.2 & $7.74 \times 10^{-5}$ & $3.23 \times 10^{-6}$ \\
0.3 & $5.00 \times 10^{-5}$ & $6.29 \times 10^{-6}$ \\
0.4 & $2.30 \times 10^{-5}$ & $9.54 \times 10^{-6}$ \\
0.5 & $2.74 \times 10^{-5}$ & $1.45 \times 10^{-5}$ \\
0.6 & $4.38 \times 10^{-5}$ & $3.27 \times 10^{-5}$ \\
0.7 & $3.87 \times 10^{-5}$ & $1.26 \times 10^{-6}$ \\
0.8 & $1.01 \times 10^{-5}$ & $1.26 \times 10^{-6}$ \\
0.9 & $3.35 \times 10^{-6}$ & $1.43 \times 10^{-7}$ \\
\hline
\end{tabular}

Table 2: Absolute errors for example 4.1 with $\mathrm{N}=2$ in domain $0<x<1$

\begin{tabular}{cc}
\hline$t$ & $\left|u(x, t)-u_{\text {approx }}(x, t)\right|$ \\
\hline 0 & 0 \\
0.1 & $2.24 \times 10^{-8}$ \\
0.2 & $3.20 \times 10^{-8}$ \\
0.3 & $4.33 \times 10^{-7}$ \\
0.4 & $7.79 \times 10^{-7}$ \\
0.5 & $8.25 \times 10^{-7}$ \\
0.6 & $2.40 \times 10^{-6}$ \\
0.7 & $4.30 \times 10^{-6}$ \\
0.8 & $5.44 \times 10^{-6}$ \\
0.9 & $4.54 \times 10^{-5}$ \\
1 & $6.21 \times 10^{-5}$ \\
\hline
\end{tabular}

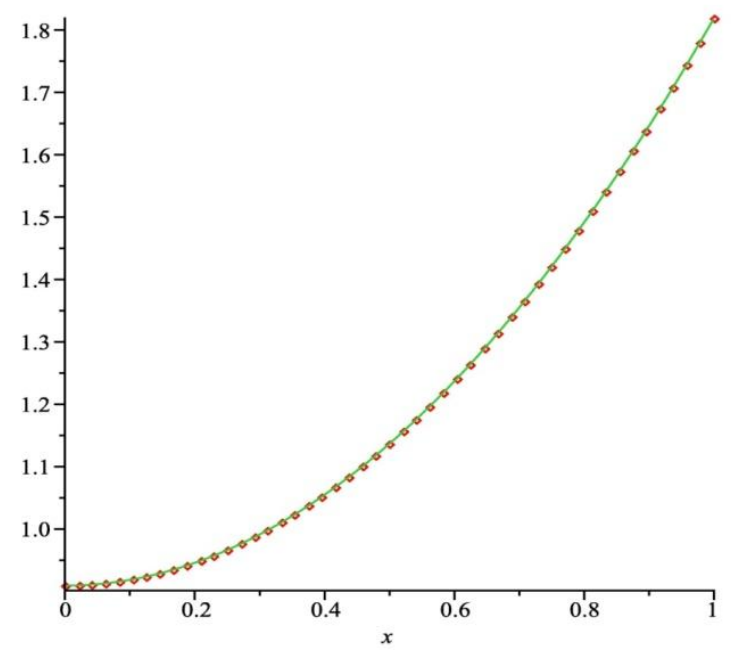

Figure 1: Approximate solution and exact solution for $u(x, 1)$ with $N=2$ for Ex. 4.1 
Example 4.2. [15] In this example, we consider the following space fractional diffusion equation:

$$
\frac{\partial u(x, t)}{\partial t}=\Gamma(1.2) x^{1.8} \frac{\partial^{1.8} u(x, t)}{\partial x^{1.8}}+3 x^{2}(2 x-1) e^{-t},
$$

with the initial condition

$u(x, 0)=x^{2}-x^{3}$,

and the boundary conditions

$u(0, t)=0, \quad u(1, t)=0, \quad$ for $t>0$.

The exact solution of this problem is $u(x, t)=x^{2}(1-x) e^{-t}$. We solved this equation by using proposed method and in Table 3 we compared our results with results of obtained in $[6,15]$. Also in Table 4 maximum absolute errors for $t=0.2,0.4, \ldots, 1,1.2, \ldots, 1.8,2$ and $0<x<1$ are reported.

Also, figure 2 shows the exact solution and approximate solution for $\mathrm{u}(\mathrm{x}, 2)$ with $N=3$. Note that the matrices $\mathbf{A}$ and $\mathbf{B}$ for this example are as follows:

$$
\begin{aligned}
A & =\left[\begin{array}{cccc}
1 & 1 & 1 & 1 \\
1 & 0.5 & -0.5039 & -1.0081 \\
1 & -0.5 & -0.5003 & 1.0013 \\
1 & -1 & 1 & -1
\end{array}\right], \\
B & =\left[\begin{array}{cccc}
0 & 0 & 0 & 0 \\
1 & 0.5 & -0.4961 & -0.9919 \\
1 & -0.5 & -0.4997 & 0.9987 \\
0 & 0 & 0 & 0
\end{array}\right] .
\end{aligned}
$$

Easily seen that $\mathbf{A}$ is non-singular matrix and the spectral radius of matrix $\mathbf{A}^{-1} \mathbf{B}$ is less than one therefore the proposed method has unique solution and is unconditionally stable.

Table 3: Comparison of absolute errors for $u(x, 2)$ for example 4.2.

\begin{tabular}{cccc}
\hline$x$ & Method [6] with m=5 & Method [15] with m=5 & present method(N=3) \\
\hline 0.0 & $2.74 \times 10^{-5}$ & 0.0 & 0.0 \\
0.1 & $4.20 \times 10^{-5}$ & $4.47 \times 10^{-6}$ & $3.15 \times 10^{-7}$ \\
0.2 & $3.76 \times 10^{-5}$ & $2.78 \times 10^{-7}$ & $4.23 \times 10^{-7}$ \\
0.3 & $8.44 \times 10^{-5}$ & $5.81 \times 10^{-6}$ & $2.94 \times 10^{-7}$ \\
0.4 & $3.27 \times 10^{-5}$ & $1.02 \times 10^{-5}$ & $5.70 \times 10^{-6}$ \\
0.5 & $3.61 \times 10^{-5}$ & $1.17 \times 10^{-5}$ & $6.28 \times 10^{-5}$ \\
0.6 & $1.94 \times 10^{-5}$ & $1.08 \times 10^{-5}$ & $1.05 \times 10^{-5}$ \\
0.7 & $2.95 \times 10^{-5}$ & $8.54 \times 10^{-6}$ & $2.43 \times 10^{-6}$ \\
0.8 & $4.92 \times 10^{-5}$ & $6.06 \times 10^{-6}$ & $8.50 \times 10^{-6}$ \\
0.9 & $2.83 \times 10^{-5}$ & $3.67 \times 10^{-6}$ & $7.68 \times 10^{-7}$ \\
1.0 & $7.73 \times 10^{-5}$ & 0.0 & 0.0 \\
\hline
\end{tabular}


Table 4: Absolute errors for example 4.2 with $\mathrm{N}=3$ in domain $0<x<1$

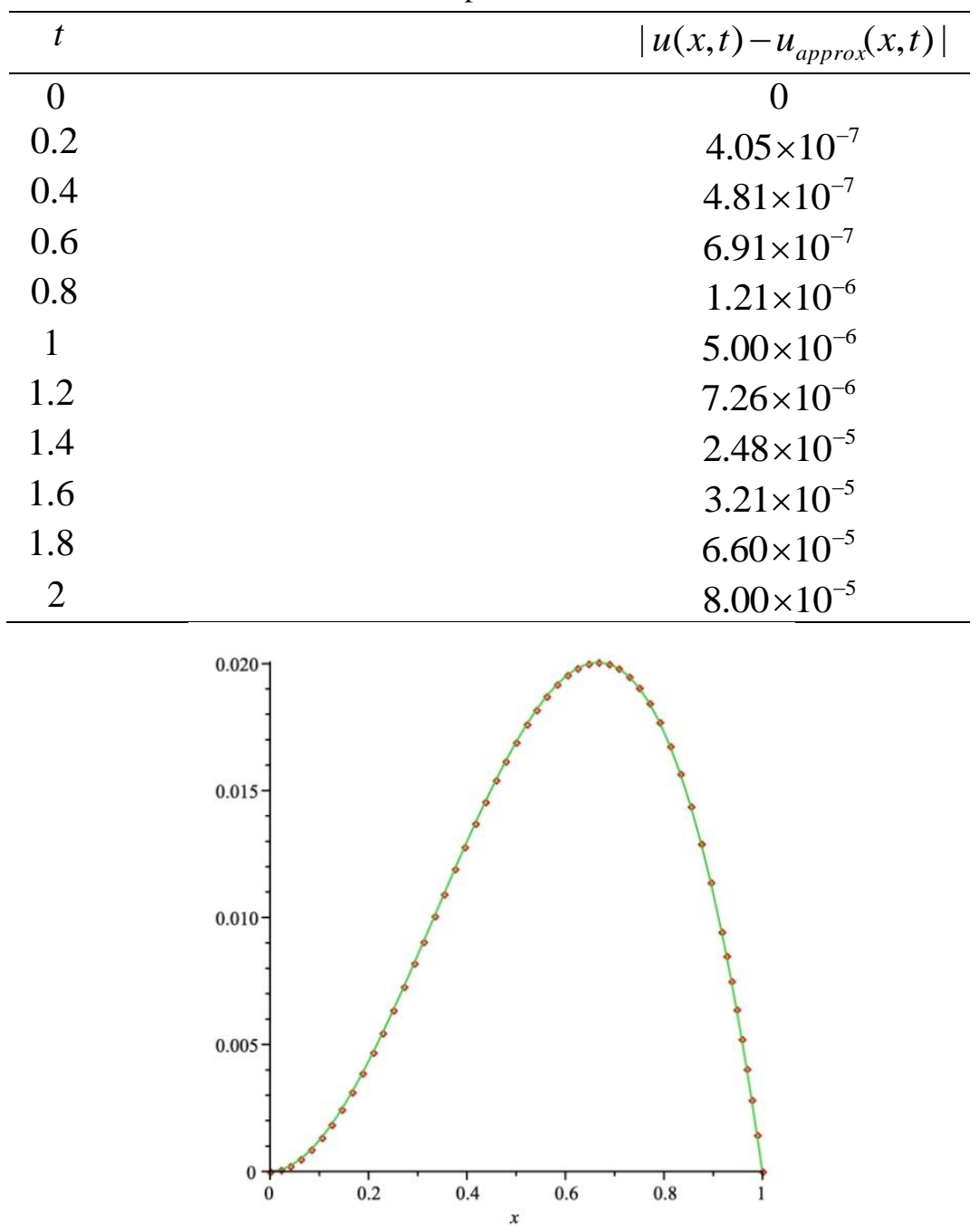

Figure 2: Approximate solution and exact solution for $u(x, 2)$ with $N=3$ for Ex. 4.2

Example 4.3. [16] Consider the problem (1) with initial condition

$$
u(x, 0)=x^{4}, 0<x<1
$$

and boundary conditions

$u(0, t)=0, u(1, t)=e^{-t}$.

Let $p(x)=\frac{1}{24} \Gamma(5-\alpha) x^{\alpha}$ and $v(x, t)=-2 e^{-t} x^{4}$. The exact solution for this problem is $u(x, t)=e^{-t} x^{4}$.

We solved this problem by applying the present method. In Table 5, we obtained the maximum errors between the exact solution and the approximate solution for different values of $\alpha$ for $N=4$ in finite domain $0 \leq x, t \leq 1$.

Also, figure 3 shows the exact solution and approximate solution for $\mathrm{u}(\mathrm{x}, 1)$ with $N=4$ for $\alpha=1.2$. The matrices $\mathbf{A}$ and $\mathbf{B}$ for this example for $\alpha=1.2$ are as follows: 


$$
\begin{aligned}
A & =\left[\begin{array}{ccccc}
1 & 1 & 1 & 1 & 1 \\
1 & 0.7071 & -0.0006 & -0.7089 & -1.003 \\
1 & 0 & -1.0002 & 0.0001 & 1.0006 \\
1 & -0.7071 & -0.000006 & 0.7072 & -1.0001 \\
1 & -1 & 1 & -1 & 1
\end{array}\right], \\
B & =\left[\begin{array}{ccccc}
0 & 0 & 0 & 0 & 0 \\
1 & 0.7071 & 0.0006 & -0.7053 & -0.9973 \\
1 & 0 & -0.9998 & -0.0001 & 0.9994 \\
1 & -0.7071 & 0.000006 & 0.7071 & -0.9998 \\
0 & 0 & 0 & 0 & 0
\end{array}\right] .
\end{aligned}
$$

Easily seen that $\mathbf{A}$ is non-singular matrix and the spectral radius of matrix $\mathbf{A}^{-\mathbf{1}} \mathbf{B}$ is less than one therefore the proposed method has unique solution and is unconditionally stable.

Note that in [16] this problem has been solved by finite difference method and splines. The maximum errors for $\alpha=1.2, \alpha=1.4, \alpha=1.5$ and $\alpha=1.8$ with $\Delta x=\frac{1}{30}$ are $0.3566 \times 10^{-3}$, $0.24616 \times 10^{-3}, 0.2067 \times 10^{-3}$ and $0.1150 \times 10^{-3}$ respectively.

Table 5: The absolute errors for $\mathrm{N}=4$ and different values of $\alpha$ for example 4.3

\begin{tabular}{ccccc}
\hline$t$ & $\alpha=1.2$ & $\alpha=1.4$ & $\alpha=1.5$ & $\alpha=1.8$ \\
\hline 0.1 & $4.89 \times 10^{-7}$ & $3.24 \times 10^{-7}$ & $2.43 \times 10^{-7}$ & $1.04 \times 10^{-7}$ \\
0.2 & $6.22 \times 10^{-7}$ & $5.68 \times 10^{-7}$ & $3.73 \times 10^{-7}$ & $1.74 \times 10^{-7}$ \\
0.3 & $7.36 \times 10^{-7}$ & $2.71 \times 10^{-6}$ & $6.15 \times 10^{-7}$ & $4.26 \times 10^{-7}$ \\
0.4 & $8.00 \times 10^{-7}$ & $4.12 \times 10^{-6}$ & $8.57 \times 10^{-7}$ & $5.91 \times 10^{-7}$ \\
0.5 & $1.38 \times 10^{-6}$ & $5.19 \times 10^{-6}$ & $1.88 \times 10^{-6}$ & $7.09 \times 10^{-7}$ \\
0.6 & $4.12 \times 10^{-6}$ & $8.06 \times 10^{-6}$ & $2.15 \times 10^{-6}$ & $2.49 \times 10^{-6}$ \\
0.7 & $7.24 \times 10^{-6}$ & $4.94 \times 10^{-5}$ & $1.67 \times 10^{-5}$ & $2.80 \times 10^{-6}$ \\
0.8 & $2.73 \times 10^{-5}$ & $5.16 \times 10^{-5}$ & $1.89 \times 10^{-5}$ & $4.55 \times 10^{-6}$ \\
0.9 & $5.40 \times 10^{-5}$ & $7.63 \times 10^{-5}$ & $4.53 \times 10^{-5}$ & $3.25 \times 10^{-5}$ \\
1 & $6.93 \times 10^{-5}$ & $8.44 \times 10^{-5}$ & $6.34 \times 10^{-5}$ & $2.43 \times 10^{-5}$ \\
\hline
\end{tabular}

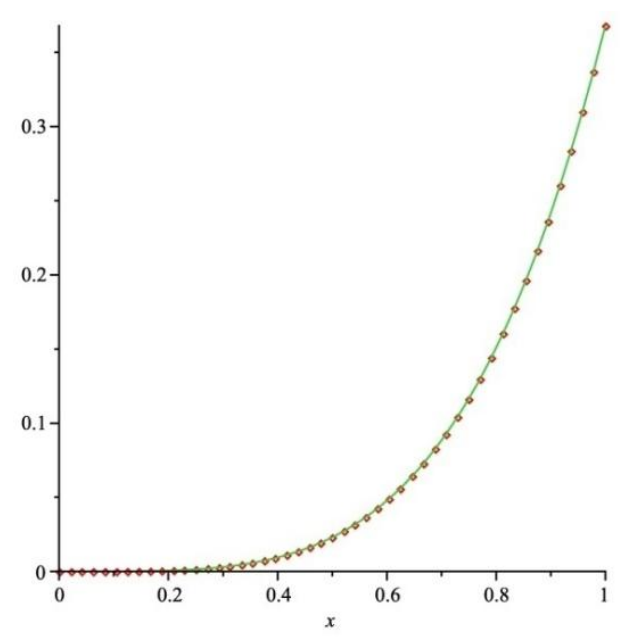

Figure 3: Approximate solution and exact solution for $u(x, 1)$ with $N=4, \alpha=1.2$. 


\section{Conclusion}

In this paper, finite difference scheme and Chebyshev collocation method have been successfully applied to find the solution of the space fractional diffusion equations. Sine using matrix form of the method is more convenient for application of collocation method, thus the matrix form of the proposed method was obtained. The results and comparison of the our proposed method and other methods indicate that this scheme is accurate and efficient approach for the solution of this problems. Proposed method for the examples of this paper was unconditionally stable but, unconditionally stable for the general case is open problem.

\section{References}

[1] G.A. Afrouzi , R.A. Talarposhti , H. Ahangar, Explicit analytical solution for a kind of time-fractional evolution equations by He's Homotopy perturbation methods, J. Math. Comput. Sci. (TJMCS) 4, 278-282 (2012).

[2] Z. Avazzadeh, Z. Beygi Rizi, F.M. Maalek Ghaini, G.B. Loghmani, A numerical solution of nonlinear parabolic-type Volterra partial integrodifferential equations using radial basis functions, Eng. Anal. Bound. Elem. 36 (2012) 881-893.

[3] R. Darzi, B. Mohammadzade, S. Mousavi, R. Beheshti, Sumudu transform method for solving fractional differential equations and fractional Diffusion-Wave equation, J. Math. Comput. Sci. (TJMCS) 6, 79-84 (2013).

[4] K. Diethelm, The analysis of fractional differential equation, Berlin, Springer-Verlag (2010).

[5] G.J. Fix, J.P. Roop, Least squares finite element solution of a fractional order two-point boundary value problem, Comput. Math. Appl. 48 (2004) 1017-1033.

[6] M.M. Khader, On the numerical solutions for the fractional diffusion equation, Commun. Nonlinear Sci. Numer. Simul. 16 (2011) 2535-2542.

[7] F. Liu, V. Anh and I. Turner, Numerical solution of the space fractional Fokker-Plank equation, J. Comput. Appl. Math. 166 (2004) 209-219.

[8] JT. Machado, V. Kiryakova and F. Mainardi, Rcent history of fractional caculus, Commun Nonlinear Sci. Numer. Simul. 16 (2011) 1140-1153.

[9] J.C. Mason, D.c. Handscomb, Chebyshev polynomials, CRC press, Boca Raton, 2003.

[10] M.M. Meerschaert, C. Tadjeran, Finite difference approximations for fractional advection-dispersion flow equations, J. Comput. Appl. Math. 172 (2004) 65-77.

[11] M.M. Meerschaert, C. Tadjeran, Finite difference approximations for two-sided space-fractional partial differential equations, Appl. Numer. Math. 56 (2006) 80-90.

[12] M.M. Meerschaert, H.P. Scheffler, C. Tadjeran, Finite difference methods for two-dimensional fractional dispersion equations, J. Comput. Phys. 211 (2006) 249-261.

[13] A. Neamaty, B. Agheli, R. Darzi, Solving fractional partial differential equation by using wavelet operational method, J. Math. Comput. Sci. (TJMCS) 7, 230 - 240 (2013).

[14] Y.Rossikhin, M. Shitikova, Application of fractional calculus for dynamic problems of solid mechanics: novel trends and recent results, Appl. Mech. Rev. 63 (2010) 1-52.

[15] A. Saadatmandi, M. Dehghan, A tau approach for solution of the space fractional diffusion equation, Compute. Math. Appl. 62 (2011) 1135-1142.

[16] E. Sousa, Numerical approximations for fractional diffusion equation via splines, Compute. Math. Appl. 62 (2011) 938944.

[17] N.H. Sweilam, M.M. Khader, A.M. Nagy, Numerical solution of twosided space-fractional wave equation using finite difference method, J. Comput. Appl. Math, 235 (2011) 2832-2841.

[18] C. Tadjeran, M.M. Meerschaert, H. Scheffler, A second-order accurate numerical approximation for the fractional diffusion equation, J. Comput. Phys. 213 (2006) 205-213.

[19] C. Tadjeran, M.M. Meerschaert, A second-order accurate numerical approximation for the two-dimensional fractional diffusion equation, J. Comput. Phys. 220 (2007) 813-823. 